\title{
Silence of miR-32-5p promotes endothelial cell viability by targeting KLF2 and serves as a diagnostic biomarker of acute myocardial infarction
}

Yunxiang Dai ${ }^{1}$, Tingguo Yan $^{2}$ and Yuming Gao ${ }^{1 *}$

\begin{abstract}
Background: MicroRNAs (miRNAs) have been investigated in various cardiovascular diseases. As a fatal disease, acute myocardial infarction (AMI) is a serious global health burden. The purpose of this study was to investigate the role of miR-32-5p in AMI patients and human umbilical vein endothelial cells (HUVECs) to explore novel diagnostic and therapeutic approaches for AMI.

Methods: A target prediction tool miRanda and the luciferase activity assay were used to confirm the interaction of miR-32-5p with Kruppel-like factor 2 (KLF2). Effect of miR-32-5p on HUVECs viability was examined using CCK-8 assay. Serum miR-32-5p expression was measured using quantitative Real-Time PCR, and its correlation with myocardial damage and endothelial injury markers and pro-inflammatory cytokines was assessed. Receiver operating characteristic (ROC) curves were used to evaluate the diagnostic value of miR-32-5p in AMI patients.

Results: miR-32-5p, as a direct regulator of KLF2, could suppress the cell proliferation of HUVECs. Serum miR-32-5p expression was elevated in AMI patients and positively correlated with the biomarker levels of myocardial damage and endothelial injury and pro-inflammatory cytokines. The area under the ROC curve for miR-32-5p was 0.949, indicating the relatively high diagnostic accuracy of miR-32-5p in AMl patients.

Conclusion: The data of this study revealed that the increased serum miR-32-5p expression serves as a candidate diagnostic biomarker of AMI, and that miR-32-5p may be involved in the myocardial damage, endothelial injury and inflammatory responses of AMI by targeting KLF2, indicating the potential of miR-32-5p as a diagnostic biomarker and molecular target to improve the treatment of AMI.
\end{abstract}

Keywords: Acute myocardial infarction, Kruppel-like factor 2, MicroRNA-32-5p, Endothelial cell, Inflammation, Diagnosis, Proliferation

\section{Introduction}

Acute myocardial infarction (AMI) a serious cardiovascular disease and a leading cause of global deaths, which contributes to the occurrence of coronary artery obstruction [1].

\footnotetext{
*Correspondence: neuron_yun@163.com

'Department of Emergency, Qingdao Jiaozhou Central Hospital, No. 29 Xuzhou Road, Qingdao 266300, Shandong, China

Full list of author information is available at the end of the article
}

There are approximately 17 million of deaths occurred annually due to cardiovascular diseases, and the AMI-related mortality accounts for about $13 \%$ [2]. It is reported that a number of patients with evolving myocardial infarction die before receiving efficient treatment [3]. Thus, early and accurate diagnosis is of great importance for the treatment of AMI. Currently, the cardiac troponin I (cTnI) is the preferred diagnostic biomarker for AMI patients, but the

C C The Author(s). 2020 Open Access This article is licensed under a Creative Commons Attribution 4.0 International License, which permits use, sharing, adaptation, distribution and reproduction in any medium or format, as long as you give appropriate credit to the original author(s) and the source, provide a link to the Creative Commons licence, and indicate if changes were made. The images or other third party material in this article are included in the article's Creative Commons licence, unless indicated otherwise in a credit line to the material. If material is not included in the article's Creative Commons licence and your intended use is not permitted by statutory regulation or exceeds the permitted use, you will need to obtain permission directly from the copyright holder. To view a copy of this licence, visit http://creativecommons.org/licenses/by/4.0/ The Creative Commons Public Domain Dedication waiver (http://creativecommons.org/publicdomain/zero/1.0/) applies to the data made available in this article, unless otherwise stated in a credit line to the data. 
elevation of cTnI is also appeared in cases with heart failure, sepsis and chronic kidney diseases, leading to the limited application of cTnI $[4,5]$. Thus, novel diagnostic biomarkers with high accuracy are necessary for AMI patients.

The disease progression of AMI is complex and has reported to related with vascular endothelial injury and inflammatory responses [6]. The normal vascular endothelium can sustain the fluid shear stress, while the injury in vascular endothelial cells triggers cardiovascular diseases [7]. To evaluate the endothelial injury degree, some biomarkers have been identified, including cTnI, heart-type fatty acid-binding protein (H-FABP) and von Willebrand factor (vWF). The sustained inflammatory response following AMI could contribute to left ventricular dysfunction and remodeling [8], leading to the strategies that attenuate inflammation become efficient therapeutic approaches to improve the clinical outcomes of AMI patients [9]. Kruppel-like factor 2 (KLF2) is a key molecule in angiogenesis and vascular formation [10] and plays a pivotal role in the regulation of endothelial proliferation in AMI [11, 12]. A study by Liu et al. has reported that KLF2 mediates the effect of miR92a on endothelial injury in AMI rats [12]. In addition, KLF2 serves an important regulator in the expression of anti-inflammatory genes, thereby involving in the pathogenesis of cardiovascular diseases [13]. These aforementioned researches inspire us to identify novel molecules that related with KLF2 to improve AMI treatment.

According to the bioinformatic prediction by miRanda (http://www.microrna.org/microrna/home.do), we found a complementary sequence of microRNA-32-5p (miR-32$5 p)$ at the 3 - untranslated region (3'-UTR) of KLF2. Numerous microRNAs (miRNAs) have been investigated in cardiovascular diseases [14, 15]. The aberrant expression of miRNAs has been determined as diagnostic biomarkers or therapeutic targets in the progression of AMI [16]. miR-32-5p has been reported to modulate the viability of vascular smooth muscle cells and play as a biomarker of coronary artery calcification [17]. In addition, the regulatory effect of miR-32-5p on inflammatory responses has been found in macrophages infected by Mycobacterium tuberculosis [18] and rats with neuropathic pain [19]. As a potential upstream regulator of KLF2, the role of miR-32$5 p$ in endothelial activation remains unclear.

To improve the diagnosis and therapy of AMI, this study sought to verify the effect of miR-32-5p on endothelial cell proliferation, assess the relationship of miR$32-5 p$ with endothelial injury and inflammation in AMI patients and evaluate its diagnostic accuracy.

\section{Materials and methods}

\section{Cell culture}

Human umbilical vein endothelial cells (HUVECs) were purchased from the Cell Bank of the Chinese Academy of Sciences (Shanghai, China) and cultured in endothelial growth medium (Gibco, CA, USA) at $37{ }^{\circ} \mathrm{C}$ in a humidified atmosphere with $5 \% \mathrm{CO}_{2}$.

\section{Patients and sample collection}

A total of 88 AMI patients were enrolled from Qingdao Jiaozhou Central Hospital between 2015 and 2017, and 50 age- and gender-matched healthy volunteers were recruited as controls at a same time period. None of the patients had received any therapy before blood sampling, and the healthy individuals had no medical history of cardiovascular diseases. The diagnosis of AMI was performed in accordance with the universal definition of myocardial infarction [20]. Venous blood was collected from the participants immediately after admission to hospital and centrifuged to isolate serum samples for subsequent analyses. The experimental procedures of this study were approved by the Ethics Committee of Qingdao Jiaozhou Central Hospital, and an informed consent was received from each participant. The demographic and clinical characteristics of the patients and healthy subjects were listed in Table 1 , and there were no statistical differences in age, gender, body mass index (BMI), history of smoking, hypertension, hyperlipidaemia, diabetes mellitus between the AMI patients and healthy controls (all $P>0.05$ ), while the AMI patients had a higher atherogenic index (AI) than the healthy individuals $(P<0.001)$.

RNA extraction and quantitative real-time PCR (qRT-PCR) Total RNA in serum and cells was extracted using TRIzol reagent (Life Technologies, Carlsbad, CA, USA) following the manufacture's protocol. The purity of RNA was evaluated using a NanoDrop 1000 spectrophotometer (Thermo Scientific, Utah, USA). Reverse transcription was performed from RNA to synthesize cDNA using a TaqMan miRNA RT Kit (Applied Biosystems, Foster City, USA).

Table 1 Demographic and clinical characteristics of the research cohort

\begin{tabular}{llll}
\hline Features & $\begin{array}{l}\text { Healthy } \\
\text { controls } \\
(n=50)\end{array}$ & $\begin{array}{l}\text { AMl patients } \\
(n=88)\end{array}$ & $P$ value \\
\hline Age (years, mean \pm SD) & $60.82 \pm 18.13$ & $60.81 \pm 17.35$ & 0.997 \\
$\begin{array}{l}\text { Gender (male/female, } \\
\% \text { of male) }\end{array}$ & $29 / 21,58.0 \%$ & $48 / 40,54.5 \%$ & 0.694 \\
BMl (kg/m², mean \pm SD) & $24.31 \pm 2.83$ & $25.25 \pm 2.88$ & 0.064 \\
Smoking (n, \%) & $26,52.0 \%$ & $46,52.3 \%$ & 0.975 \\
Hypertension (n, \%) & $28,56.0 \%$ & $47,53.4 \%$ & 0.769 \\
Hyperlipidaemia (n, \%) & $25,50.0 \%$ & $46,52.3 \%$ & 0.797 \\
Diabetes mellitus (n, \%) & $14,28.0 \%$ & $27,30.7 \%$ & 0.740 \\
Al (mean \pm SD) & $1.84 \pm 0.56$ & $6.49 \pm 1.68$ & $<0.001$ \\
\hline
\end{tabular}

BMI Body mass index, Al Atherogenic index 
The expression of miR-32-5p and mRNA of KLF2 was estimated by qRT-PCR using the SYBR green I Master Mix kit (Invitrogen, Carlsbad, CA, USA) on the 7300 RealTime PCR System (Applied Biosystems, USA) with the following reaction conditions: $95^{\circ} \mathrm{C}$ for $10 \mathrm{~min}, 95^{\circ} \mathrm{C}$ for $30 \mathrm{~s}, 60^{\circ} \mathrm{C}$ for $20 \mathrm{~s}, 72^{\circ} \mathrm{C}$ for $15 \mathrm{~s}$, a total of 40 cycles. The relative expression levels of miR-32-5p and KLF2 were calculated using the $2^{-\Delta \Delta C t}$ method and normalized to U6 and GAPDH, respectively.

\section{Luciferase activity assay}

The complementary sequence of miR-32-5p at the 3'-UTR of KLF2 was predicted by the miRanda (http://www.microrna.org/microrna/home.do). To verify the interaction between miR-32-5p and KLF2, a luciferase reporter assay was performed. The 3'-UTR of KLF2 containing the putative binding sites of miR-32-5p was cloned and inserted into the luciferase reporter vector pmiR-REPORT (Life Technologies, USA). The reporter vectors carried wild type (WT) or mutant type (MT) of KLF2 3'-UTR were cotransfected into HUVECs with miR-32-5p mimic or miR32-5p inhibitor (GenePharma, Shanghai, China) using Lipofectamine 3000 (Invitrogen, Carlsbad, CA, USA) following the protocols of manufacturers. Twenty-four hours later, the relative luciferase activity was measured using a Luciferase 1000 Assay System (Promega, USA).

\section{CCK-8 assay}

To verify whether miR-32-5p was involved in the regulation of endothelial cell proliferation, a Cell Counting Kit-8 (CCK-8; Beyotime, Nantong, China) was used to evaluate the proliferation of HUVECs. HUVECs were transfected with miR-32-5p mimic, miR-32-5p inhibitor or the corresponding negative controls (mimic NC and inhibitor NC) (GenePharma, Shanghai, China) using Lipofectamine 3000 (Invitrogen, Carlsbad, CA, USA) as per the manufacturer's instruction. At $48 \mathrm{~h}$ after cell transfection, HUVECs with a cell density of $3 \times 10^{3}$ cell/well were seeded into 96-well plates and supplemented with CCK-8 reagent at 0, 24, 48, $72 \mathrm{~h}$ with further $4 \mathrm{~h}$ of incubation. The absorbance at $450 \mathrm{~nm}$ of the cell culture was measured by a microplate reader (BioTek Instruments, VT, USA).

\section{Enzyme-linked immunosorbent assay (ELISA)}

The serum levels of myocardial damage and endothelial injury biomarkers (cTnI, H-FABP and vWF) and proinflammatory cytokines (IL-1 $\beta$, IL- 6 and TNF- $\alpha$ ) were examined using the ELISA kit (RayBiotech, GA, USA) following the manufacturers' instruction.

\section{Statistical analysis}

Data were presented as mean $\pm \mathrm{SD}$ and analyzed using SPSS 18.0 software (SPSS Inc., Chicago, IL) and GraphPad Prism 5.0 software (GraphPad Software, Inc., USA).
Comparisons between groups were analyzed using Student's $t$ test and one-way ANOVA. Correlations of miR$32-5 p$ with endothelial injury markers and proinflammatory cytokines were performed using Pearson correlation analysis. Receiver operating characteristic (ROC) curves and area under the curve (AUC) were used to evaluate the diagnostic accuracy of miR-32-5p. A value of $P<0.05$ was considered of statistically significant.

\section{Results}

\section{KLF2 is a direct target of miR-32-5p}

By using the bioinformatic prediction tool miRanda, a complementary sequence of miR-32-5p was found in the 3'-UTR of KLF2 (Fig. 1a). A subsequent luciferase assay was carried out to confirm their interaction. By cell transfection, we confirmed that the expression of miR$32-5 p$ in HUVECs could be successfully upregulated by miR-32-5p mimic and downregulated by miR-32-5p inhibitor (both $P<0.001$, Fig. 1b). The relative luciferase results shown in Fig. 1c indicated that the relative luciferase activity in cells transfected with WT 3 '-UTR of KLF2 was significantly inhibited by the overexpression of miR-32-5p, while was enhanced by the silence of miR-32-5p (all $P<0.05)$. By contrast, there were no luciferase activity changes in cells with MT 3'-UTR after upregulating or downregulating the levels of miR-32-5p. Thus, KLF2 was demonstrated as a direct target gene of miR-32-5p in HUVECs.

\section{Effect of miR-32-5p on cell viability of HUVECs}

Considering the pivotal role of KLF2 in endothelial cell proliferation, this study further investigated the effect of miR-32-5p on HUVECs viability using CCK- 8 assay. As shown in Fig. 1d, the overexpression of miR-32-5p by miR-32-5p mimic could suppress, whereas the knockdown of miR-32-5p by miR-32-5p inhibitor could enhance the cell proliferation in HUVECs (all $P<0.05$ ).

\section{Serum expression of miR-32-5p and KLF2 in patients with AMI}

Eighty-eight patients with AMI were enrolled in this study to further confirm the role of miR-32-5p in AMI. By qRT-PCR, we found that the serum mRNA of KLF2 was decreased in AMI patients compared with the healthy controls $(P<0.01$, Fig. 2a). Inversely, a significant increase was observed in the expression of miR-32-5p in AMI patients when compared to the healthy volunteers $(P<0.01$, Fig. 2 b). A further correlation assay results shown in Fig. 2c revealed that serum expression of miR$32-5 p$ was negatively correlated with the serum mRNA levels of KLF2 $(\mathrm{r}=-0.813, P<0.001)$. 

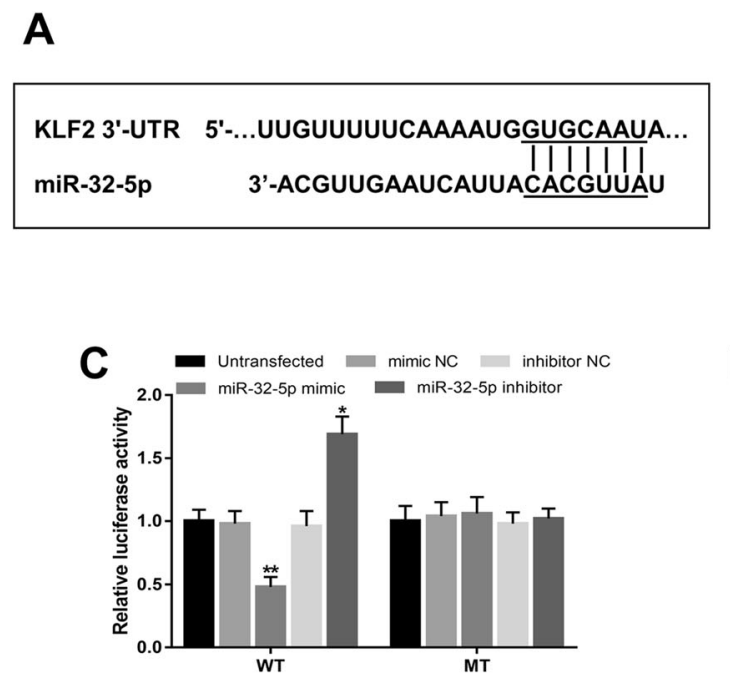
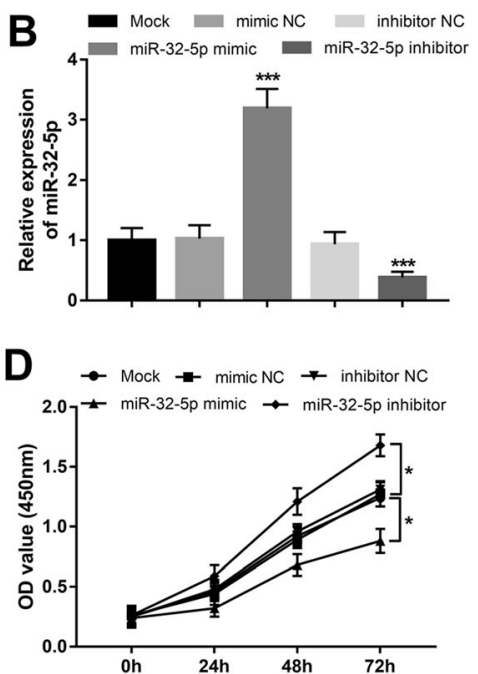

Fig. 1 Interaction of miR-32-5p with KLF2 and its effect on HUVECS proliferation. $\mathbf{a}$. The binding sequence of miR-32-5p in the 3'-UTR of KLF2. $\mathbf{b}$. Expression of miR-32-5p was successfully upregulated by the miR-32-5p mimic, and was downregulated by the miR-32-5p inhibitor. $\mathbf{c}$. The relative luciferase activity in HUVECs with overexpression or knockdown of miR-32-5p. d. HUVECs proliferation was promoted by the downregulation of miR-32-5p, but was inhibited by the overexpression of miR-32-5p. No significant difference was found in miR-32-5p expression, relative luciferase activity and cell proliferation between mock group and mimic NC or inhibitor NC group, which excluded the effect of transfection operations on the detected results. KLF2, Kruppel-like factor 2; $3^{\prime}$-UTR, 3'-untranslated region; NC, negative control; WT, wild type; MT, mutant type; ${ }^{*} P<0.05$, ${ }^{* *} P<0.01,{ }^{* * *} P<0.001$

\section{Correlation of miR-32-5p with Al, biomarkers of} myocardial damage and endothelial injury and inflammation in AMI patients

Considering the reported relationship between miR-32$5 \mathrm{p}$ and atherosclerosis, the values of AI, which were calculated based on levels of serum total cholesterol, highand low-density lipoprotein, were used to represent the degrees of atherosclerosis in AMI patients [21]. Serum levels of cTnI, H-FABP and vWF and concentration of IL-1 $\beta$, IL- 6 and TNF- $\alpha$ were measured and listed in Table 2 to reflect the status of myocardial damage, endothelial injury and inflammatory response. According to a correlation analysis, the positive correlations of miR-32$5 \mathrm{p}$ were demonstrated with AI $(r=0.694, P<0.001)$, cTnI $(r=0.650, P<0.001)$, H-FABP $(r=0.570, P=$ $0.004)$, vWF $(r=0.649, P=0.001)$, IL-1 $\beta(r=0.633, P=$ $0.012)$, IL-6 $(r=0.514, P=0.017)$ and TNF- $\alpha(r=0.654$, $P=0.008)$.

\section{Diagnostic value of serum miR-32-5p expression in patients with AMI}

cTnI, as the preferred indicator for the diagnosis of AMI, was proven to be positively correlated with the serum expression of miR-32-5p (Table 2). As shown in Fig. 3a, the ROC curve constructed based on cTnI showed a high diagnostic accuracy, a result manifested by an AUC value of 0.992. For the serum miR-32-5p, we also found the considerable diagnostic accuracy of miR-32-5p with an AUC of 0.949 (Fig. 3b). At the optimal cutoff value of 1.385, the sensitivity and specificity of serum miR-32-5p were respectively 92.0 and $84.0 \%$.

\section{Discussion}

AMI is one of the leading causes of death worldwide and needs efficient strategies for early diagnosis and therapy. This study sought to explore a novel miRNA molecule that might play a critical role in the pathogenesis of AMI.
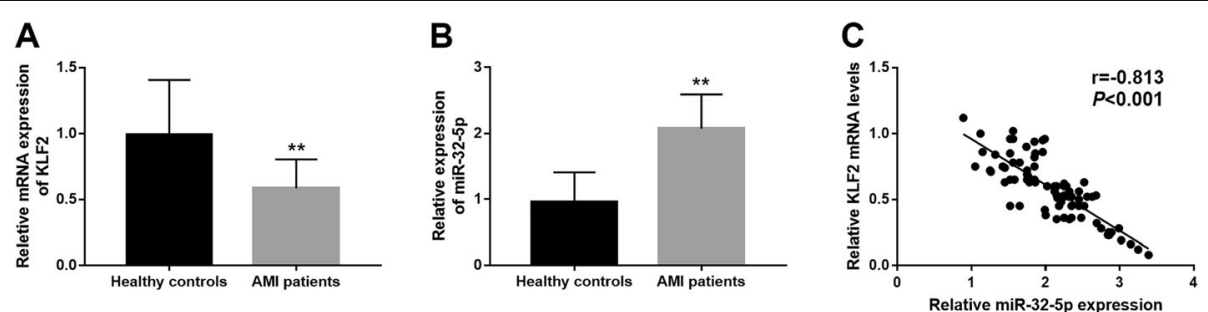

Fig. 2 Serum expression of KLF2 and miR-32-5p in patients with AMI. a. Serum mRNA expression of KLF2 was decreased in AMI patients compared with the healthy controls. b. Serum miR-32-5p expression was higher in AMl patients than that in the healthy controls. c. Serum miR32-5p levels were negatively correlated with serum mRNA levels of KLF2 $(r=-0.813, P<0.001)$. ${ }^{* *} P<0.01$ 
Table 2 Correlation of miR-32-5p with Al, serum levels of myocardial damage and endothelial injury markers and proinflammatory cytokines

\begin{tabular}{llll}
\hline Indicators & Values & \multicolumn{2}{l}{ miR-32-5p } \\
\cline { 3 - 4 } & & $r$ & $P$ \\
\hline Al & $6.49 \pm 1.68$ & 0.694 & $<0.001$ \\
CTnl $(\mathrm{ng} / \mathrm{mL})$ & $4.11 \pm 1.16$ & 0.650 & $<0.001$ \\
H-FABP $(\mathrm{ng} / \mathrm{mL})$ & $38.43 \pm 10.05$ & 0.570 & 0.004 \\
VWF $(\mathrm{ng} / \mathrm{mL})$ & $53.28 \pm 7.64$ & 0.649 & 0.001 \\
IL-1ß $(\mathrm{pg} / \mathrm{mL})$ & $20.15 \pm 5.95$ & 0.633 & 0.012 \\
IL-6 $(\mathrm{pg} / \mathrm{mL})$ & $61.32 \pm 10.18$ & 0.514 & 0.017 \\
TNF-a $(\mathrm{pg} / \mathrm{mL})$ & $51.69 \pm 9.63$ & 0.654 & 0.008 \\
\hline AlAtherogic &
\end{tabular}

Al Atherogenic index, cTnl Cardiac troponin I, H-FABP Heart-type fatty acidbinding protein; vWF Von Willebrand factor, IL Interleukin, TNF Tumor necrosis factor

By bioinformatics and luciferase activity assay, miR-32-5p was predicted and determined as an upstream regulator of KLF2 in HUVECs. Cell viability assay showed that the proliferation of HUVECs was suppressed by the overexpression of miR-32-5p, but was enhanced by the knockdown of miR-32-5p. Further clinical research data indicated that the upregulated expression levels of miR32-5p in AMI patients were negatively correlated with the increased expression levels of KLF2. The serum miR-32$5 p$ levels were positively correlated with the levels of myocardial damage and endothelial injury markers (cTnI, HFABP and vWF), pro-inflammatory cytokines (IL-1 $\beta$, IL-6 and TNF- $\alpha$ ) and AI. For the clinical significance evaluation, the ROC curve based on serum expression of miR$32-5 p$ showed a relatively high diagnostic accuracy for miR-32-5p in patients with AMI.

Endothelial function is significantly impaired during the progression of AMI, which could be indicated by the inhibited cell viability [22]. For the treatment of AMI, the endothelial activation has been considered as an important signal for the therapeutic efficacy [23], and the explorations of novel molecules that serve as candidate therapeutic targets were performed by investigating the changes in endothelial function [12]. miRNAs are involved in the regulation of cell proliferation, and some members of them have been determined with pivotal roles in AMI pathogenesis by modulating endothelial cell viability $[12,22]$. For example, Huang et al. reported that miR-103a expression was elevated in AMI patients and involved in the pathogenesis of AMI by regulating the endothelial function, a result manifested by the inhibiting effect of miR-103a on cell proliferation of HUVECs [24]. Bayoumin et al. gave evidence for miR532 as a regulator of cardiac endothelial cell proliferation and deduced that miR-532 had a cardioprotective effect against AMI-associated ischemic heart diseases [22]. Yu et al. investigated the role of miR-133a in AMI patients following radical surgery for gastric cancer, and found that miR-133a expression was increased in AMI patients and the silence of miR-133a in HUVECs led to enhanced cell proliferation, indicating the critical role of miR-133a in the endothelial injury process after AMI [25]. These aforementioned literatures implied us to identify novel functional miRNAs in AMI progression by focusing on their relationship with endothelial cell function.

KLF2 has been identified as a regulator of endothelial activation with promoting effect on HUVECs viability [12, 26]. This study used bioinformatics prediction to find that miR-32-5p was a regulator of KLF2 by binding its $3^{\prime}$ UTR. Previous literatures have reported that regulatory effect of miR-32-5p on cell proliferation in cervical cancer cells [27] and cardiac fibroblast [28]. However, there was no report regarding to the relationship of miR-32-5p with endothelial cell viability. In the present study, the cell viability results showed that the cell proliferation of HUVECs
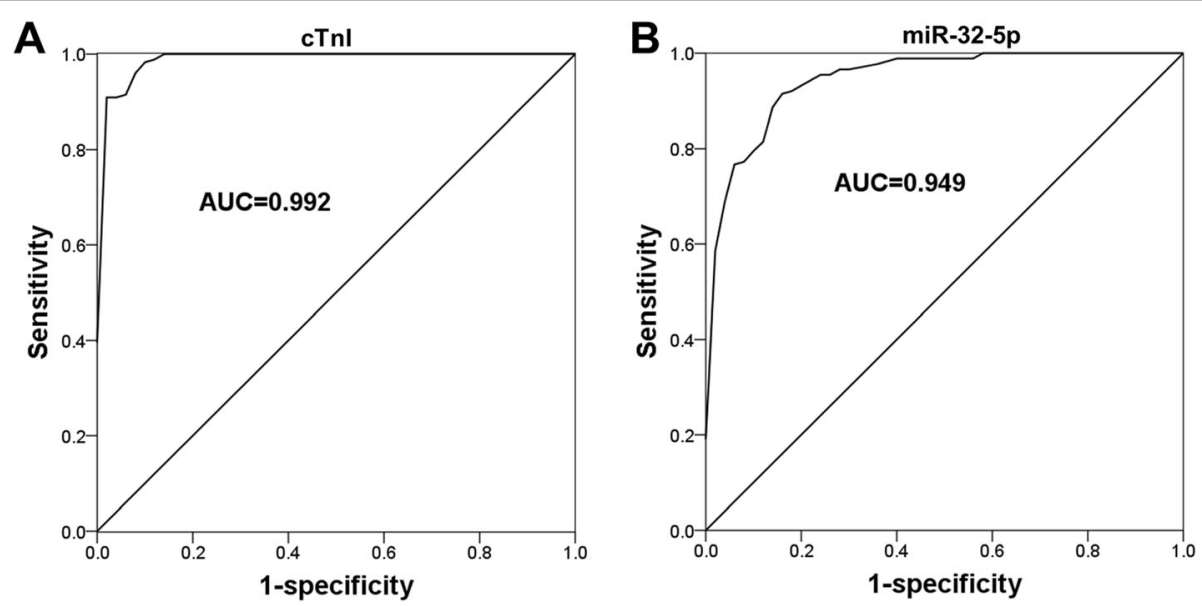

Fig. 3 ROC curves for AMl patients based on cTnl and miR-32-5p. a. A ROC curve based on serum cTnl (AUC=0.992). b. A ROC curve based on serum levels of miR-32-5p (AUC $=0.949)$ 
could be inhibited by the overexpression of miR-32-5p, while was promoted by the knockdown of miR-32-5p, which indicated that miR-32-5p might be involved in endothelial cell viability by targeting KLF2. Furthermore, the expression examination results revealed a significant increase in the expression of miR-32-5p in patients with AMI compared with healthy controls. It is reported that miR-32-5p may serve a critical role in the progression of atherosclerosis by regulating arterial calcification [17, 29]. In this study, the AI values of AMI patients were significantly high, and we found that the serum levels of miR$32-5 \mathrm{p}$ was positively correlated with the AI values, indicating that miR-32-5p might be involved in the development of AMI by regulating the arterial calcification. The subsequent correlation analysis found the positive correlation between miR-32-5p and the endothelial injury biomarkers. Thus, we considered that the elevated expression of miR32-5p might be involved in the endothelial injury in the pathogenesis of AMI.

After the occurrence of AMI, the left ventricular dysfunction and remodeling can be promoted by the sustained inflammatory responses. KLF2 has been found to regulate some key molecules or signaling involved in inflammation [30, 31]. As a regulator of KLF2, miR-32-5p has been found to contribute to inflammatory responses in macrophages infected by Mycobacterium tuberculosis [18] and rats with neuropathic pain [19]. Thus, the relationship of miR-32-5p was further analyzed with the serum levels of inflammatory cytokines in AMI patients. The statistical correlation analysis data showed that miR-32-5p was positively correlated with IL- $1 \beta$, IL- 6 and TNF- $\alpha$, indicating that miR-32-5p might participate the inflammatory responses through regulating KLF2. However, whether miR-32-5p has regulatory effect on the inflammatory responses in AMI was not assessed in this study, warrant further investigations.

There are lots of AMI patients died before receiving hospital therapy, and early diagnosis remains one of the approaches to reduce AMI mortality. The dysregulation of miRNAs in various diseases has attracted increasing attention on their diagnostic value [32]. In prostate cancer patients, the deregulated expression of miR-32-5p has been identified as a diagnostic biomarker [33]. Considering the upregulation of miR-32-5p in AMI patient, this study constructed a ROC curve based on miR-32-5p expression levels and showed that serum expression of miR-32-5p had relatively high diagnostic accuracy to distinguish AMI patients from healthy controls. Thus, the elevated serum miR-32-5p might serve as a diagnostic biomarker of AMI. However, the diagnostic potential results of miR-32-5p might be limited by the small sample size of this study. Thus, further investigations with a larger study cohort are needed to confirm the clinical significance and functional role of miR-32-5p in AMI.
Taken together, miR-32-5p is a direct regulator of KLF2 and may be involved in the endothelial injury and inflammatory responses in the pathogenesis of AMI. The elevated serum miR-32-5p expression in AMI patients may serve as a candidate diagnostic biomarker for the screening of AMI patients. This study provides a novel insight into the diagnosis and pathogenesis of AMI, and the strategies to inhibit miR-32-5p may have potentials to improve AMI treatment.

\begin{abstract}
Abbreviations
3'-UTR: 3'-untranslated region; Al: Atherogenic index; AMI: Acute myocardial infarction; AUC: Area under the curve; BMI: Body mass index; CCK-8: Cell Counting Kit-8; CTnl: cardiac troponin I; H-FABP: Heart-type fatty acid-binding protein; HUVECs: Human umbilical vein endothelial cells; KLF2: Kruppel-like factor 2; miRNAs: microRNAs; MT: Mutant type; NC: Negative control; qRTPCR: quantitative Real-Time PCR; ROC: Receiver operating characteristic; VWF: von Willebrand factor; WT: Wild type
\end{abstract}

\section{Acknowledgements}

Not applicable.

\section{Authors' contributions}

YD and YG designed and performed the clinical analyses and wrote the manuscript. TY performed the cell experiments and analyzed cell data. The authors read and approved the final manuscript.

\section{Funding}

Not applicable.

\section{Availability of data and materials}

All data generated or analyzed during this study are included in this published article.

\section{Ethics approval and consent to participate}

The experimental procedures of this study were approved by the Ethics Committee of Qingdao Jiaozhou Central Hospital, and an informed consent was received from each participant.

\section{Consent for publication}

Written informed consent for publication was obtained from each participant.

\section{Competing interests}

The authors declare that they have no competing interests.

\section{Author details}

'Department of Emergency, Qingdao Jiaozhou Central Hospital, No. 29 Xuzhou Road, Qingdao 266300, Shandong, China. ${ }^{2}$ Department of cardiovascular medicine, Anqiu People's Hospital, Weifang 262100,

Shandong, China.

Received: 21 November 2019 Accepted: 26 February 2020

Published online: 03 March 2020

\section{References}

1. Boateng S, Sanborn T. Acute myocardial infarction. Dis Mon. 2013;59:83-96.

2. Shafei $A E$, Ali MA, Ghanem HG, et al. Mesenchymal stem cell therapy: $A$ promising cell-based therapy for treatment of myocardial infarction. J Gene Med. 2017:19:12.

3. Shibata T, Kawakami S, Noguchi T, et al. Prevalence, clinical features, and prognosis of acute myocardial infarction attributable to coronary artery embolism. Circulation. 2015:132:241-50.

4. Keller T, Zeller T, Peetz D, et al. Sensitive troponin I assay in early diagnosis of acute myocardial infarction. N Engl J Med. 2009:361:868-77.

5. Finsterer J, Stollberger C, Krugluger W. Cardiac and noncardiac, particularly neuromuscular, disease with troponin-T positivity. Neth J Med. 2007:65:28995. 
6. Hernandez-Resendiz S, Munoz-Vega M, Contreras WE, et al. Responses of endothelial cells towards ischemic conditioning following acute myocardial infarction. Cond Med. 2018;1:247-58.

7. Tuenter A, Selwaness M, Arias Lorza A, et al. High shear stress relates to intraplaque haemorrhage in asymptomatic carotid plaques. Atherosclerosis. 2016;251:348-54

8. Garikipati VNS, Verma SK, Jolardarashi D, et al. Therapeutic inhibition of miR375 attenuates post-myocardial infarction inflammatory response and left ventricular dysfunction via PDK-1-AKT signalling axis. Cardiovasc Res. 2017; 113:938-49.

9. Ong SB, Hernandez-Resendiz S, Crespo-Avilan GE, et al. Inflammation following acute myocardial infarction: multiple players, dynamic roles, and novel therapeutic opportunities. Pharmacol Ther. 2018;186:73-87.

10. Sangwung $P$, Zhou G, Nayak L, et al. KLF2 and KLF4 control endothelial identity and vascular integrity. JCI Insight. 2017;2:e91700.

11. Dekker RJ, van Soest S, Fontijn RD, et al. Prolonged fluid shear stress induces a distinct set of endothelial cell genes, most specifically lung Kruppel-like factor (KLF2). Blood. 2002;100:1689-98.

12. Liu H, Li G, Zhao W, Hu Y. Inhibition of MiR-92a may protect endothelial cells after acute myocardial infarction in rats: role of KLF2/4. Med Sci Monit. 2016;22:2451-62

13. $\mathrm{Xu} Y, \mathrm{Xu}$ S, Liu P, et al. Suberanilohydroxamic Acid as a Pharmacological Kruppel-Like Factor 2 Activator That Represses Vascular Inflammation and Atherosclerosis. J Am Heart Assoc. 2017;6:12.

14. Ding S, Huang $H, X u Y, Z$, hu H, Zhong C. MiR-222 in cardiovascular diseases: physiology and pathology. Biomed Res Int. 2017;2017:4962426.

15. Vacante F, Denby L, Sluimer JC, Baker AH. The function of miR-143, miR-145 and the MiR-143 host gene in cardiovascular development and disease. Vasc Pharmacol. 2019;112:24-30.

16. Liu X, Yuan L, Chen F, et al. Circulating miR-208b: a potentially sensitive and reliable biomarker for the diagnosis and prognosis of acute myocardial infarction. Clin Lab. 2017;63:101-9.

17. Liu J, Xiao X, Shen $Y$, et al. MicroRNA-32 promotes calcification in vascular smooth muscle cells: implications as a novel marker for coronary artery calcification. PLoS One. 2017;12:e0174138.

18. Zhang ZM, Zhang AR, Xu M, Lou J, Qiu WQ. TLR-4/miRNA-32-5p/FSTL signaling regulates mycobacterial survival and inflammatory responses in mycobacterium tuberculosis-infected macrophages. Exp Cell Res. 2017;352: 313-21

19. Yan T, Zhang F, Sun C, et al. miR-32-5p-mediated Dusp5 downregulation contributes to neuropathic pain. Biochem Biophys Res Commun. 2018;495: 506-11.

20. Thygesen K, Alpert JS, Jaffe AS, et al. Third universal definition of myocardial infarction. J Am Coll Cardiol. 2012;60:1581-98.

21. Kazemi T, Hajihosseini M, Moossavi M, Hemmati M, Ziaee M. Cardiovascular risk factors and Atherogenic indices in an Iranian population: Birjand east of Iran. Clin Med Insights Cardiol. 2018;12:1179546818759286.

22. Bayoumi AS, Teoh JP, Aonuma T, et al. MicroRNA-532 protects the heart in acute myocardial infarction, and represses prss23, a positive regulator of endothelial-to-mesenchymal transition. Cardiovasc Res. 2017;113:1603-14.

23. Rakic M, Persic $V$, Kehler $T$, et al. Possible role of circulating endothelial cells in patients after acute myocardial infarction. Med Hypotheses. 2018;117:426.

24. Huang L, Li L, Chen X, Zhang H, Shi Z. MiR-103a targeting Piezo1 is involved in acute myocardial infarction through regulating endothelium function. Cardiol J. 2016;23:556-62.

25. Yu J, Cao X, Zheng Y, Yan L, Wang J. Abnormal expression of miR133a in patients with acute myocardial infarction following radical surgery for gastric cancer and the underlying mechanism. Mol Med Rep. 2018;18:50239

26. Manavski Y, Abel T, Hu J, et al. Endothelial transcription factor KLF2 negatively regulates liver regeneration via induction of activin a. Proc Natl Acad Sci U S A. 2017;114:3993-8.

27. Liu YJ, Zhou HG, Chen LH, et al. MiR-32-5p regulates the proliferation and metastasis of cervical cancer cells by targeting HOXB8. Eur Rev Med Pharmacol Sci. 2019;23:87-95.

28. Shen J, Xing W, Liu R, Zhang Y, Xie C, Gong F. MiR-32-5p influences high glucose-induced cardiac fibroblast proliferation and phenotypic alteration by inhibiting DUSP1. BMC Mol Biol. 2019;20:21.
29. Cao J, Chen L, Zhong X, et al. miR32-5p promoted vascular smooth muscle cell calcification by upregulating TNFalpha in the microenvironment. BMC Immunol. 2020;21:3

30. Jha P, Das H. KLF2 in Regulation of NF-kappaB-Mediated Immune Cell Function and Inflammation. Int J Mol Sci. 2017;18:E2383.

31. Shi J, Zhou LR, Wang XS, et al. KLF2 attenuates bleomycin-induced pulmonary fibrosis and inflammation with regulation of AP-1. Biochem Biophys Res Commun. 2018;495:20-6.

32. McGuire A, Brown JA, Kerin MJ. Metastatic breast cancer: the potential of miRNA for diagnosis and treatment monitoring. Cancer Metastasis Rev. 2015;34:145-55.

33. Daniel R, Wu Q, Williams V, Clark G, Guruli G, Zehner Z. A Panel of MicroRNAs as Diagnostic Biomarkers for the Identification of Prostate Cancer. Int J Mol Sci. 2017;18:6.

\section{Publisher's Note}

Springer Nature remains neutral with regard to jurisdictional claims in published maps and institutional affiliations.
Ready to submit your research? Choose BMC and benefit from:

- fast, convenient online submission

- thorough peer review by experienced researchers in your field

- rapid publication on acceptance

- support for research data, including large and complex data types

- gold Open Access which fosters wider collaboration and increased citations

- maximum visibility for your research: over $100 \mathrm{M}$ website views per year

At BMC, research is always in progress.

Learn more biomedcentral.com/submissions 